PROCEEDINGS OF THE

AMERICAN MATHEMATICAL SOCIETY

Volume 125, Number 7, July 1997, Pages 1945-1952

S 0002-9939(97)03794-5

\title{
LINEARLY COMPACT ALGEBRAIC LIE ALGEBRAS AND COALGEBRAIC LIE COALGEBRAS
}

\author{
BIENVENIDO CUARTERO, JOSÉ E. GALÉ, AND ARKADII M. SLINKO \\ (Communicated by Roe W. Goodman)
}

\begin{abstract}
It is proved that if the dual Lie algebra of a Lie coalgebra is algebraic, then it is algebraic of bounded degree. This result is an analog of the D.Radford's result for associative coalgebras.
\end{abstract}

\section{MAin RESUlts}

In 1974 D. Radford [8] showed that if the dual algebra $A$ of an associative coalgebra $C$ is algebraic it is necessarily algebraic of bounded degree. From the point of view that some recent results provide this fact can be explained as follows. Endowed with the weak linear topology the dual algebra $A$ becomes a topological linearly compact algebra $[9,10]$ and since linearly compact algebras are Baire algebras the result of the type "algebraic implies algebraic of bounded degree" is true for them even in alternative or Jordan case [4].

In this paper we are looking from this point of view at Lie algebras and Lie coalgebras and obtain similar results. Let us recall that an element $x$ of a Lie algebra $L$ over a field $K$ is called algebraic if there exists a polynomial $f(t) \in K[t]$ depending on $x$ such that $f(\operatorname{ad} x)=0$. The degree of $x$, which by definition is the minimal degree of polynomials annihilating ad $x$, will be denoted by $\operatorname{deg}(x)$. For convenience, if $x$ is not algebraic we consider that $\operatorname{deg}(x)=\infty$. A Lie algebra $L$ is called algebraic if every element $x \in L$ is algebraic. If in addition degrees of all elements of $L$ are bounded from above by some positive integer, $L$ is called algebraic of bounded degree.

Theorem 1. Every algebraic linearly compact Lie algebra is algebraic of bounded degree.

As we mentioned the analog of the Radford's result will follow from here.

Received by the editors August 31, 1995 and, in revised form, January 25, 1996.

1991 Mathematics Subject Classification. Primary 17B99.

Key words and phrases. Lie coalgebra, dual Lie algebra, algebraic Lie algebra of bounded degree.

The research of the first two authors has been partially supported by the Project PS090-0120, DGICYT, Spain.

This paper was written when the third author visited Universities of Oviedo and Zaragoza in January-February 1995. It is his great pleasure to express his gratitude to both Departments of Mathematics for the hospitality and DGICYT (PS 90-0120) and The University of Oviedo for the financial support. 
Theorem 2. If the dual Lie algebra $L=C^{*}$ of a Lie coalgebra $C$ is algebraic, it is algebraic of bounded degree.

Some proofs make heavy use of deep results of E.I. Zelmanov for Lie PI-algebras developed in $[12,13,14]$.

\section{TOPOlOGicAl LEMmas}

All algebras and coalgebras are considered over an arbitrary field $K$ which is always discrete.

Definition 1. A topological vector space $E$ over a discrete field $K$ is said to be locally balanced if it has a basic system of zero neighborhoods $V$ such that $K V=V$.

In particular, if $E$ has a base of zero neighborhoods consisting of linear subspaces (i.e., if $E$ is linearly topologized) then it is locally balanced.

Lemma 1. Let $E$ be a locally balanced topological vector space over $K$. Then $\lim _{i} \lambda_{i} z_{i}=0$ whenever $\lim _{i} z_{i}=0$.

Proof. It is a straightforward consequence of the existence of a locally balanced neighborhood base.

The following lemma, which lies in the basis of the main topological argument, is an analog of a well-known result of Kaplansky [6]. It appeared previously in [3] but it is not currently easily available and we give a proof of it for convenience of the reader.

Lemma 2. Let $E$ be a locally balanced topological vector space over $K$. For $1 \leq$ $n \leq N$, let $\left(x_{i, n}\right)_{i \in I}$ be a finite set of $N$ nets in $E$ converging to linearly independent vectors $x_{1}, \ldots, x_{N} \in E$. Suppose that for every index $i \in I$ there is a linear combination $y_{i}=\sum_{n=1}^{N} \lambda_{i, n} x_{i, n}$ such that the net $\left(y_{i}\right)_{i \in I}$ converges to some $y$. Then for each $n$ the net $\left(\lambda_{i, n}\right)_{i \in I}$ converges in $K$.

Proof. It is known ([2, Chap.1 $\S 2$, Ex.14] that the subspace $E_{N}$, which is spanned by $\left\{x_{1}, \ldots, x_{N}\right\}$ in $E$, must be discrete and that the mapping

$$
\left(\lambda_{1}, \ldots, \lambda_{N}\right) \in K^{N} \mapsto \sum_{n=1}^{N} \lambda_{n} x_{n} \in E_{N}
$$

is a homeomorphism. Noting that

$$
\begin{aligned}
& \sum_{n=1}^{N}\left(\lambda_{i, n}-\lambda_{j, n}\right) x_{n} \\
= & \sum_{n=1}^{N} \lambda_{i, n} x_{i, n}+\sum_{n=1}^{N} \lambda_{i, n}\left(x_{n}-x_{i, n}\right)-\sum_{n=1}^{N} \lambda_{j, n} x_{j, n}+\sum_{n=1}^{N} \lambda_{j, n}\left(x_{j, n}-x_{n}\right) \\
= & y_{i}-y_{j}+\sum_{n=1}^{N} \lambda_{i, n}\left(x_{n}-x_{i, n}\right)+\sum_{n=1}^{N} \lambda_{j, n}\left(x_{j, n}-x_{n}\right),
\end{aligned}
$$

we obtain according to Lemma 1 that $\lim _{i, j}\left(\lambda_{i, n}-\lambda_{j, n}\right)=0$ for $1 \leq n \leq N$, i.e., there is an index $i_{n}$ such that $\lambda_{i, n}$ is constant for $i \geq i_{n}$. 
Let $L$ be a linearly compact Lie algebra over $K$ and $R(L)$ be its multiplication algebra generated by all operators ad $x, x \in L$, with topology defined by a base of neighborhoods of zero $\Omega(V)=\{\omega \in R(L) \mid L \omega \subseteq V\}$, where $V$ is an arbitrary element from the base of neighborhoods of zero in $L$.

Lemma 3. Endowed with the topology introduced, $R(L)$ is a linearly topologized topological algebra.

Proof. We will only check that multiplication is continuous. Let $\omega_{1}, \omega_{2}$ be arbitrary elements of $R(L)$. We will show that for a given $V$, if $\omega_{1}^{\prime}$ and $\omega_{2}^{\prime}$ are sufficiently close to $\omega_{1}$ and $\omega_{2}$, respectively, then

$$
\omega_{1}^{\prime} \omega_{2}^{\prime}-\omega_{1} \omega_{2} \in \Omega(V)
$$

Suppose that

$$
\omega_{2}=\sum_{i} \operatorname{ad} x_{i, 1} \ldots \operatorname{ad} x_{i, n_{i}}
$$

and $n=\max _{i} n_{i}$.

By Lemma 1 of [9] there exists a neighborhood $W$ of zero in $L$ such that $[L, W] \subseteq$ $V$. Using this lemma $n$ times we can find a neighborhood $W$ of zero in $L$ such that

$$
[\ldots[W, \underbrace{L], \ldots, L}_{k}] \subseteq V
$$

for $k=1, \ldots, n$. Intersecting $W$ with $V$, if necessary, we can also consider that $W \subseteq V$.

Suppose now that $\omega_{i}^{\prime}-\omega_{i} \in \Omega(W), i=1,2$, and write

$$
\omega_{1}^{\prime} \omega_{2}^{\prime}-\omega_{1} \omega_{2}=\left(\omega_{1}^{\prime}-\omega_{1}\right)\left(\omega_{2}^{\prime}-\omega_{2}\right)+\omega_{1}\left(\omega_{2}^{\prime}-\omega_{2}\right)+\left(\omega_{1}^{\prime}-\omega_{1}\right) \omega_{2} .
$$

Apply the operator (2) to $L$. Then $L\left(\omega_{1}^{\prime}-\omega_{1}\right)\left(\omega_{2}^{\prime}-\omega_{2}\right) \subseteq W$ and $L \omega_{1}\left(\omega_{2}^{\prime}-\omega_{2}\right) \subseteq W$ by the definition of $\Omega(W)$. Also

$$
L\left(\omega_{1}^{\prime}-\omega_{1}\right) \omega_{2} \subseteq W \omega_{2} \subseteq \sum_{i=1}^{n}[\ldots[W, \underbrace{L], \ldots, L}_{k}] \subseteq V .
$$

So, due to (2), inclusion (1) is true and therefore the multiplication in $R(L)$ is continuous.

Corollary 1. The mappings $\operatorname{ad}^{n}: x \mapsto(\operatorname{ad} x)^{n}$ are continuous.

Proof. As we have seen in the proof of the lemma, for an arbitrary neighborhood $V$ of zero in $L$ there exists a neighborhood $W$ of zero in $L$ such that $[L, W] \subseteq V$. In other words it means that $\operatorname{ad}(W) \subseteq \Omega(V)$ and the mapping $x \mapsto \operatorname{ad} x$ is continuous. But since the product in $R(L)$ is continuous, all mappings ad ${ }^{n}$ are continuous.

Lemma 4. Let $L$ be a linearly compact algebra over $K$. Then the sets

$$
A_{n}(L)=\{x \in L \mid \operatorname{deg}(x) \leq n\}
$$

are closed.

Proof. Let $\left(x_{i}\right)_{i \in I}$ be a net in $A_{n}(L)$ converging to $x$. Then the set $\left\{\left(\operatorname{ad} x_{i}\right)^{m} \mid 1 \leq\right.$ $m \leq n\}$ is linearly dependent for every $i \in I$. As the mappings ad ${ }^{m}$ are continuous by Lemma 3 the nets $\left(\left(\operatorname{ad} x_{i}\right)^{m}\right)_{i \in I}$ converge to $(\operatorname{ad} x)^{m}$ for all $1 \leq m \leq n$. By Lemma 2 elements $(\operatorname{ad} x)^{m}$ for $1 \leq m \leq n$ are linearly dependent and therefore an annihilating polynomial of degree not greater than $n$ exists for ad $x$ and $x \in$ $A_{n}(L)$. 


\section{Algebraic lemmas}

Let $K$ be an arbitrary field.

Definition 2. A linear operator $\phi: E \rightarrow E$ acting on a vector space $E$ over $K$ is said to be weakly algebraic (or locally algebraic, see [7]) if for every $x \in E$ there exists a polynomial $f(t) \in K[t]$ depending on $x$ such that $x f(\phi)=0$. If degrees of all such polynomials have the least upper bound $n$ the operator $\phi$ is said to be weakly algebraic of bounded degree $n$.

The following lemma belongs to the mathematical folklore but we will need a bit stronger version of it than in [7, Lemma 14, p.41] [5, Proposition 1], [1, Chap.4, p.141].

Lemma 5. Let $E$ be a vector space over $K$ and let $\phi$ be a weakly algebraic linear operator on $E$ of bounded degree $n$. Then $\phi$ is algebraic of some degree which is bounded from above by some positive integer $N$ depending only on $n$.

Proof. In a usual way we can consider $E$ as a $K[t]$-module letting

$$
x \cdot f(t)=x f(\phi) .
$$

Let $f_{x}(t)$ be a polynomial of degree $\leq n$ such that $x \cdot f_{x}(t)=0$. It is well-known that if

$$
f_{x}(t)=\pi_{1}^{l_{1}}(t) \ldots \pi_{s}^{l_{s}}(t)
$$

is the factorization of $f_{x}(t)$ into irreducible factors, then

$$
x=x_{1}+\cdots+x_{s},
$$

where $x_{i} \cdot \pi_{i}^{l_{i}}(t)=0$. For every $\pi(t)$ irreducible, define

$$
E_{\pi(t)}=\left\{x \in E \mid x \cdot \pi^{n}(t)=0\right\} .
$$

Then $E=\sum E_{\pi(t)}$. Moreover, there will be no more than $n$ of $E_{\pi(t)}$ which are nonzero; otherwise we can find an element of $E$ whose minimal annihilating polynomial is of degree greater than $n$.

Suppose now that only $E_{\pi_{1}(t)}, \ldots, E_{\pi_{m}(t)}$ are nonzero. We see that polynomial

$$
F(t)=\pi_{1}^{n}(t) \ldots \pi_{m}^{n}(t)
$$

annihilates all elements of $E$ and his degree is less than $N=n^{n}$. Therefore $\phi$ is algebraic of degree $\leq N$.

In the sequel we will need to apply Zelmanov's results which appeared in [12][14]. But first we present them in the form of the following two theorems in which they will be ready for use.

Theorem 3 (Zelmanov). Let $L=L\left\langle x_{1}, \ldots, x_{m}\right\rangle$ be a free $m$-generated algebra in a variety of Lie algebras defined by a homogeneous polynomial identity of degree $n$. Let $k$ be a natural number. Then there exist numbers $h(m, n)$ and $d(m, n, k)$ such that any commutator on $X=\left\{x_{1}, \ldots, x_{m}\right\}$ of length greater than or equal to $d(m, n, k)$ can be represented as a linear combination of commutators of the same length and the same degree in each variable such that each of them contains a subcommutator of the form

$$
[\tau, \rho, \ldots, \rho]=\tau(\operatorname{ad} \rho)^{k}
$$

for some commutators $\tau, \rho$, and the length of $\rho$ is less than $h(m, n)$. 
Proof. Take the Zelmanov's function $h(m, n)$ of [14, Theorem 3] and generate an ideal $I$ in $L$ by all elements of the form (3). Then the quotient-algebra $L / I$ is nilpotent of some index $d$ by Theorem 3 of [14]. Since $L$ is a free algebra in a variety defined by a homogeneous polynomial identity, this number $d$ can serve as $d(m, n, k)$.

Theorem 4 (Zelmanov). Suppose that a Lie algebra $L$ is generated by a finite subset $X=\left\{x_{1}, \ldots, x_{m}\right\}$, and assume that

(1) L satisfies a polynomial identity of degree $n$;

(2) For an arbitrary commutator $\rho$ on $X$ of length not greater than $h(m, n)$ the operator ad $\rho$ is algebraic of degree not greater than $k$.

Then $L$ is finite-dimensional of dimension bounded from above by a number $D(m, n, k)$ which depends only on $m, n, k$.

Proof. According to the previous theorem commutators of length less than $d(m, n, k)$ form a subset which spans $L$ linearly. Since there are only a finite numbers of such commutators, the theorem is proved.

Lemma 6. Let $L$ be a Lie algebra, $I$ be an ideal of $L$, and suppose that for some element $a \in L$ all elements of the coset $a+I$ are algebraic of degree bounded from above by $n$. Then all elements of $I$ are algebraic of degree bounded from above by a number which depends only on $n$.

Proof. We will first note that the subalgebra $I_{1}$ of $L$ generated by $a$ and $I$ satisfies a polynomial identity. Let $S_{n}\left(x_{1}, \ldots, x_{n}\right)$ be the standard non-associative polynomial with the fixed arrangement of brackets on each monomial. First of all, we note that for all $y \in I, x \in L$

$$
S_{n+1}\left(x \operatorname{ad}(a+y), \ldots, x \operatorname{ad}(a+y)^{n+1}\right)=0 .
$$

It is a generalized polynomial identity in a sense that it contains a fixed element $a$ of L as "generalized" coefficient. We use Theorem 7 of $[15, \mathrm{p} .16]$ to get an ordinary polynomial identity. Indeed, according to this theorem, which is true also for generalized polynomials, the ideal $I$ satisfies the complete linearization of the homogeneous component of (4) of maximal total degree in $x$ and $y$. But this homogeneous component is an ordinary polynomial; so is its complete linearization. Since $I_{1}^{2} \subseteq I$, it is clear that $I_{1}$ also satisfies a certain polynomial identity whose degree will be denoted as $k$.

We will derive now some relations. Let $z$ now be an arbitrary element of $I$. Since the element $a+z$ is algebraic of degree $n$ we can write

$$
\operatorname{ad}(a+z)^{n}=\sum_{i=1}^{n-1} \alpha_{i}(z) \operatorname{ad}(a+z)^{n-i}
$$

for some $\alpha_{1}(z), \ldots, \alpha_{n-1}(z) \in K$. In particular for $z=0$ we get

$$
\operatorname{ad}(a)^{n}=\sum_{i=1}^{n-1} \alpha_{i}(0) \operatorname{ad}(a)^{n-i} .
$$

So we can rewrite (5) in the form

$$
\operatorname{ad}(z)^{n}=P(\operatorname{ad} a, \operatorname{ad} z)+\ldots,
$$


where $P(s, t)$ is a homogeneous polynomial of degree $n$ whose each monomial contains both $s$ and $t$ and dots substitute a polynomial in ad $a$ and $\operatorname{ad} z$ of total degree less than $n$.

Let us fix now an arbitrary element $x \in I$. We are going to prove that the dimension of the subalgebra $L_{x}=\langle a, x\rangle$ generated by elements $a$ and $x$ is bounded from above by a number which does not depend on $x$. It is sufficient to show that commutators on $\{a, x\}$ of length not greater than $d(2, k, n)$ span the subalgebra. Let $u$ be a commutator in $a, x$, and $v$ be an arbitrary element of $L_{x}$. During the proof we shall write $u \equiv v$ if $u-v$ can be represented as a linear combination of commutators in $a, x$ whose total degree in $a$ and $x$ is less than that of $u$.

In fact, if $\nu$ is a commutator of length $d(2, k, n)$, then according to Theorem 3 it can be represented as a linear combination of commutators each containing a subcommutator of the form

$$
\nu=[\tau, \rho, \ldots, \rho]=\tau(\operatorname{ad} \rho)^{n} .
$$

If $\rho=a$ we use (6) to get $\nu \equiv 0$. If $\rho$ contains both $a$ and $x$ then $\rho \in I$ and we use (7) to get

$$
\nu=\tau(\operatorname{ad} \rho)^{n} \equiv \tau P(\operatorname{ad} a, \operatorname{ad} \rho),
$$

which means that $\nu \equiv 0$ because at least one $\rho$ was replaced by $a$ and the total degree of $\rho$ in $a$ and $x$ is greater than 1 . In the remaining case $\rho=x$ we again use (7) to get

$$
\nu=\tau(\operatorname{ad} x)^{n} \equiv \tau P(\operatorname{ad} a, \operatorname{ad} x) .
$$

The right-hand side has in this case the same total degree as the left-hand-side but every commutator which is a summand of it has more $a$ 's than the left-hand-side. This process now can be again applied to all commutators of the right-hand-side. And again, if (6) and (7) are not applicable, the number of $a$ 's will grow. Since total degree remains constant, eventually (6) or (7) will be applicable and we will get $\nu \equiv 0$. This finishes the proof of finite-dimensionality of $L_{x}$.

Finally consider now the Lie subalgebra $R_{x}=\langle\operatorname{ad} a, \operatorname{ad} x\rangle$ of the Lie algebra $R(L)^{(-)}$generated by ad $a$ and $\operatorname{ad} x$. It is a homomorphic image of the subalgebra $L_{x}$ and therefore the dimension of $R_{x}$ is not greater than that of $L_{x}$. Since ad $\rho=$ $\operatorname{ad}(a+\rho)-\operatorname{ad} a$ it is spanned by algebraic elements of degree $n$ and therefore its associative enveloping algebra is also finite-dimensional and it is clear that its dimension is bounded from above with some number not depending on $x$. Hence $x$ is algebraic with the degree less than this number and $I$ is of bounded degree.

Lemma 7. Let $L$ be an algebraic Lie algebra containing an ideal I of finite codimension whose elements are algebraic of bounded degree. Then $L$ is algebraic of bounded degree.

Proof. Let us note first that $L$ satisfies a polynomial identity. Suppose that $\operatorname{dim} L / I$ $=n$ and $\operatorname{deg}(x) \leq m$ for all $x \in I$. Then $I$ will satisfy the following identity:

$$
S_{m+1}\left(x \operatorname{ad} y, \ldots, x(\operatorname{ad} y)^{m+1}\right)=0,
$$

$x \in L, y \in I$, and where $S_{m+1}$ is the standard non-associative polynomial with the fixed brackets arrangement on each monomial. To get an identity on the whole algebra $L$ we notice that

$$
u S_{n+1}\left(\operatorname{ad} v_{1}, \ldots, \operatorname{ad} v_{n+1}\right) \in I
$$


for all $u, v_{1}, \ldots, v_{n+1} \in L$ and substitute this expression instead of $y$ in (9). Let $N$ be the degree of the identity obtained.

We are going to prove now that elements of $L$ are weakly algebraic of bounded degree. By Lemma 5 it will be enough to prove the lemma. Let $x, y \in L$ be two arbitrary elements of $L$. Then for some basis $\left\{e_{1}, \ldots, e_{n}\right\}$ modulo $I$ we can write $x=\sum_{i=1}^{n} \alpha_{i} e_{i}+u, y=\sum_{i=1}^{n} \beta_{i} e_{i}+v$, where $\alpha_{i}, \beta_{i} \in K ; u, v \in I$. Therefore the subalgebra generated by $x$ and $y$ is contained in the subalgebra

$$
L_{u, v}=\left\langle e_{1}, \ldots, e_{n}, u, v\right\rangle .
$$

Let $h=h(n+2, N)$, where $h$ is the Zelmanov's function mentioned in the formulation of Theorem 4. It is clear that degrees of all commutators on the set $\left\{e_{1}, \ldots, e_{n}, u, v\right\}$ of length $\leq h$ will be bounded from above by a number $M$ which does not depend on $u$ and $v$. Therefore

$$
\operatorname{dim}_{K} L_{u, v} \leq D(n+2, N, M)
$$

also does not depend on $u$ and $v$. It implies that $L$ is weakly algebraic and hence algebraic.

\section{Proof of Theorem 1}

We can combine now all algebraic and topological bits and pieces together. Let $L$ be an algebraic Lie algebra over an infinite field $K$. Then the sets

$$
A_{n}(L)=\{x \in L \mid \operatorname{deg}(x) \leq n\}
$$

by Lemma 4 are closed and since $L$ is algebraic it is a countable union of these sets. As linearly compact spaces are Baire spaces [11] they cannot be represented by a countable number of closed subsets without interior points. This means that some $A_{n}(L)$ contains an interior point, say $a$. This point belongs to $A_{n}(L)$ together with an open neighborhood $W$. But in [9] it was shown that $L$ has a neighborhood base consisting of ideals of finite codimension. Thus we can consider that $W=a+I \subseteq$ $A_{n}(L)$, where $I$ is an ideal of finite codimension. Now by Lemma 6 the ideal $I$ consists of elements of bounded degree and by Lemma 7 the same can be said for the whole algebra $L$. The theorem is proved.

\section{REFERENCES}

[1] Y. Bakhturin, A. Mikhalev, V. Petrogradsky and M. Zaicev, Infinite dimensional Lie Superalgebras. Walter de Gruyter and Co, Berlin-New York, 1992.

[2] N. Bourbaki, Espaces Vectoriels Topologiques (Chap. 1 et 2). Hermann, Paris, 1966. MR 34:3277

[3] B. Cuartero and J.E. Galé, Locally PI-algebras over valued fields, In: Aportaciones Matematicas en Memoria del Profesor V.M.Onieva, Santander, Universidad de Cantabria, 1991, pp. 137-145. MR 92h:46112

[4] B. Cuartero and J.E. Galé, Bounded Degree of Algebraic Topological Algebras, Communications in Algebra, 22(1) (1994), 329-337. MR 94m:17002

[5] B. Cuartero, J.E. Galé, A. Rodriguez Palacios and A. Slinko, Bounded Degree of Weakly Algebraic Topological Lie Algebras, Manuscripta Math. 81 (1993), 129-139. MR 94k:17008

[6] I. Kaplansky, Topological Methods in Valuation Theory, Duke Math. J. 14 (1947), 527-541. MR 9:172f

[7] I. Kaplansky, Infinite abelian groups. University of Michigan Press, 1954. MR 16:444g

[8] D.E. Radford, Coalgebraic Coalgebras, Proc. Amer. Math. Soc. 45 (1974), 11-18. MR 50:9942

[9] A. Slinko, Local Finiteness of Coalgebraic Lie Coalgebras, Communications in Algebra, 23(3) (1995), 1165-1170. CMP 95:08 
[10] A. Slinko, Linearly Compact Algebras and Coalgebras, New Zealand Math. J., to appear. CMP 96:15

[11] S. Warner, Linearly Compact Rings and Modules, Math Ann. 197 (1972), 29-43. MR 45:6874

[12] E.I. Zelmanov, Nil Rings and Periodic Groups. KMS Lecture Notes in Mathematics, Korean Math. Soc., Seoul, 1992. MR 94c: 16027

[13] E.I. Zelmanov, On Periodic Compact Groups, Israel J. Math., 77 (1992), 83-95. MR 94e:20055

[14] E.I. Zelmanov, On the Restricted Burnside Problem. In: Proceedings of the International Congress of Mathematicians, Kyoto, Japan, 1990, The Mathematical Society of Japan, 1991, pp. 395-402. MR 93d:20076

[15] K.A. Zhevlakov, A.M. Slinko, I.P. Shestakov, A.I. Shirshov, Rings that are nearly associative, Academic Press, New York - London, 1982, pp. 371. MR 83i:17001

Departamento de Matemáticas, Universidad de Zaragoza, 50009 Zaragoza, Spain

E-mail address: cuartero@cc.unizar.es

Departamento de Matemáticas, Universidad de Zaragoza, 50009 Zaragoza, Spain

E-mail address: gale@cc.unizar.es

Department of Mathematics, University of Auckland, Private Bag 92019 Auckland, NeW ZEALAND

E-mail address: a.slinko@auckland.ac.nz 